\title{
Validating the Emotional Interaction Style-Assessment in children with attachment problems and an intellectual disability, and their parents
}

J.H. de Visser ${ }^{1}$, M.C. Kasius ${ }^{2}$, P.F.A. de $\mathrm{Nijs}^{3}$ \& E. Hofstra

1 Medical student 2 De Banjaard, Child and Adolescent Psychiatric Institute 3 Department of Child and Adolescent Psychiatry/Psychology

Contact: j.h.devisser@erasmusmc.nl

\section{BACKGROUND}

Children with an intellectual disability (ID) are more vulnerable to attachment problems. Several studies show the impact of attachment problems. Still little is known about attachment problems in children with an ID and there are no standard instruments in use. The Marschak Interaction Method (MIM) is an observation tool for assessing parent-child interaction. The purpose of this study was to validate the Emotional Interaction Style-Assessment (EIS), a scoring method to facilitate the interpretation of the MIM, in children with ID.

\section{METHODS}

The convergent validity of the EIS was investigated by examining its correlation with the Parenting Stress Questionnaire (PSQ), and its ability to differentiate the quality of the parentchild interaction between parents and children with attachment problems and ID (13 dyads), and parents and children with neither attachment problems nor an ID (20 dyads). First the MIM was conducted, and then the parent or caregiver completed the $\mathrm{PSQ}$.

\begin{tabular}{|c|c|c|c|c|c|}
\hline STRUCTURING AND CHALLENGING & $\begin{array}{l}\text { Non- } \\
\text { existent }\end{array}$ & Inadequate & Variable & Adequate & Good \\
\hline $\begin{array}{l}\text { 1. Parent's comprehensive ability to structure the } \\
\text { situation relative to child's developmental level } \\
\text { 2. Child's general co-operation and ability to } \\
\text { focus on the situation }\end{array}$ & $1 \mathrm{abc}$ & $\begin{array}{l}2 \mathrm{ab} \\
2 \mathrm{abc}\end{array}$ & $\begin{array}{l}3 \mathrm{ab} \\
3 \mathrm{abc}\end{array}$ & $\begin{array}{l}4 \\
4\end{array}$ & \\
\hline $\begin{array}{l}\text { Engagement: EMOTIONAL RECIPROCITY and } \\
\text { INITIATIVE }\end{array}$ & & & & & \\
\hline $\begin{array}{l}\text { 3. Parent's emotional reciprocity and initiative } \\
\text { 4. Child's emotional reciprocity and initiative }\end{array}$ & $\begin{array}{l}1 \mathrm{ab} \\
1 \mathrm{abc}\end{array}$ & $\begin{array}{l}2 a b \\
2 a b c\end{array}$ & $\begin{array}{l}3 a \mathrm{ab} \\
3 \mathrm{abc}\end{array}$ & $\begin{array}{l}4 \\
4\end{array}$ & $\begin{array}{l}5 \\
5\end{array}$ \\
\hline $\begin{array}{ll}\text { NURTURE } \\
\end{array}$ & & & & & \\
\hline $\begin{array}{l}\text { 5. Parental nurture } \\
\text { 6. Child's response to nurture }\end{array}$ & $\begin{array}{l}1 \mathrm{ab} \\
1 \mathrm{abc}\end{array}$ & $\begin{array}{l}2 a b \\
2 a b c\end{array}$ & $\begin{array}{l}3 a b \\
3 a b c\end{array}$ & $\begin{array}{l}4 \\
4\end{array}$ & $\begin{array}{l}5 \\
5\end{array}$ \\
\hline PLAYFULLNESS & & & & & \\
\hline $\begin{array}{l}\text { 7. Parent's playfullness } \\
\text { 8. Child's playfullness }\end{array}$ & $\begin{array}{l}1 \mathrm{ab} \\
1 \mathrm{abc}\end{array}$ & $\begin{array}{l}2 a b \\
2 a b c\end{array}$ & $\begin{array}{l}3 \mathrm{ab} \\
3 \mathrm{abc}\end{array}$ & $\begin{array}{l}4 \\
4\end{array}$ & $\begin{array}{l}5 \\
5\end{array}$ \\
\hline REPRESENTATION QUALITY & 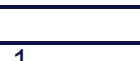 & 2 & 3 & 4 & 5 \\
\hline
\end{tabular}

\section{RESULTS}

\begin{tabular}{lcc}
\hline Table 2. Sample demographics & & \\
\hline Variable & $\begin{array}{c}\text { Population group }(n=13) \\
M(S D ; \text { range or \%) }\end{array}$ & $\begin{array}{c}\text { Control group }(n=20) \\
M(S D ; \text { range or \%) }\end{array}$ \\
\hline Age of mothers, years & $39,0(8,1 ; 27-52)$ & $37,5(5,5 ; 27-49)$ \\
Age of children, years & $9,7(2,1 ; 5-12)$ & $7,8(3,1 ; 4-14)$ \\
Dutch or western ethnicity of mothers, $\boldsymbol{n}(\%)$ & $9(69,2)$ & $20(100)$ \\
Dutch or western ethnicity of children, $\boldsymbol{n}(\%)$ & $7(53,8)$ & $20(100)$ \\
Girls, $\boldsymbol{n}(\%)$ & $8(61,5)$ & $11(55,0)$ \\
Mothers' education & $0^{*}$ & 0 \\
$\quad$ Less than high school, $n(\%)$ & $7(53,8)^{*}$ & $4(20,0)$ \\
$\quad$ High school and mbo, $n(\%)$ & $3(23,1)^{*}$ & $16(80,0)$ \\
$\quad$ College, $n(\%)$ & $12(92,3)$ & $20(100)$ \\
Biological parent, $\boldsymbol{n}(\%)$ & $68,3(11,5 ; 48-82)$ & \\
IQ of children &
\end{tabular}

Note. Means and standard deviations or percentages for sample demographics

Three missing values

No data available

\begin{tabular}{|c|c|c|c|c|c|c|}
\hline \multirow[b]{2}{*}{ Validity measure } & \multicolumn{6}{|c|}{ EIS scale } \\
\hline & $\begin{array}{l}\text { Structuring } \\
\text { and } \\
\text { Challenging }\end{array}$ & $\begin{array}{l}\text { Emotional } \\
\text { Reciprocity } \\
\text { and Initiative }\end{array}$ & Nurture & Playfullness & $\begin{array}{l}\text { Represent- } \\
\text { ation Quality }\end{array}$ & Tota \\
\hline $\begin{array}{ll}\text { Parenting } & \text { Stress } \\
\text { Questionnaire } & \\
\end{array}$ & & & & & & \\
\hline $\begin{array}{l}\text { Problems } \\
\text { caregiver-child } \\
\text { relationship }\end{array}$ & -.46 & $-.55^{* *}$ & $-.38^{*}$ & -29 & -.25 & $-.46^{\circ}$ \\
\hline Child rearing problems & $-.54^{* *}$ & $-.61^{* *}$ & $-.43^{*}$ & $-.37^{*}$ & -30 & $-.52^{\circ}$ \\
\hline Depressive mood & $-.43^{*}$ & $-.566^{* 1}$ & $-.48^{* *}$ & -.33 & -.19 & -.47 \\
\hline Role limitations & -.27 & -.34 & $-.36^{*}$ & -.11 & -.09 & -.28 \\
\hline Health problems & -.15 & -.19 & .01 & .06 & -.13 & -.11 \\
\hline Total & $-.45^{* *}$ & $-.54^{* *}$ & $-.38^{*}$ & -.25 & -.24 & $-.44^{*}$ \\
\hline
\end{tabular}

Table 4. Analyses of covariance of Emotional Interaction Style-Assessment (EIS) Total score, and

\begin{tabular}{|c|c|c|c|c|}
\hline Measure & $F(1,29)$ & $p$ & $\eta_{p}^{2}$ & $\eta^{2}$ \\
\hline \multicolumn{5}{|l|}{ EIS Total } \\
\hline Covariate: ethnicity child & 126 & .726 & .004 & .003 \\
\hline Covariate: ethnicity mother & .154 & .698 & .005 & .003 \\
\hline Group (study population vs. comparison) & 16.810 & .000 & .367 & .365 \\
\hline \multicolumn{5}{|l|}{ EIS Structuring and Challenging } \\
\hline Covariate: ethnicity child & .283 & .599 & .010 & .006 \\
\hline Covariate: ethnicity mother & .107 & .746 & .004 & .002 \\
\hline Group (study population vs. comparison) & 14.670 & .001 & .336 & .333 \\
\hline \multicolumn{5}{|l|}{ EIS Emotional Reciprocity and Initiative } \\
\hline Covariate: ethnicity child & .219 & .643 & .007 & .004 \\
\hline Covariate: ethnicity mother & .021 & .887 & .001 & .000 \\
\hline Group (study population vs. comparison) & 24.741 & .000 & .460 & .458 \\
\hline \multicolumn{5}{|l|}{ EIS Nurture } \\
\hline Covariate: ethnicity child & .337 & .566 & .011 & .010 \\
\hline Covariate: ethnicity mother & .004 & .949 & .000 & .000 \\
\hline Group (study population vs. comparison) & 4.211 & .049 & .127 & .126 \\
\hline \multicolumn{5}{|l|}{ EIS Playfulness } \\
\hline Covariate: ethnicity child & 178 & .676 & .006 & .005 \\
\hline Covariate: ethnicity mother & .132 & .719 & .005 & .003 \\
\hline Group (study population vs. comparison) & 9.731 & .004 & .251 & .249 \\
\hline \multicolumn{5}{|l|}{ EIS Representation Quality } \\
\hline Covariate: ethnicity child & .495 & .487 & .017 & .013 \\
\hline Covariate: ethnicity mother & 3.533 & .070 & .109 & .092 \\
\hline Group (study population vs. comparison) & 5.359 & .028 & .156 & .140 \\
\hline
\end{tabular}

Note. F-test, p-value, partial eta squared and eta squared for analyses of covariance of Emotional Interaction Style-Assessment (EIS) Total score, and subscales

\section{CONCLUSION}

The correlation between the PSQ and the EIS, and the ability of the EIS to differentiate between both groups supports the convergent and construct validity of the EIS. This shows that the EIS captures the quality of the parent-child interaction, so that the EIS will be a valuable instrument to use for assessment of the parent-child interaction in children with ID

\section{ACKNOWLEDGEMENTS}

Juliana van Stolbergschool, Kapelle, The Netherlands De Banjaard, The Hague, The Netherlands

Saara Salo, Finland

\section{REFERENCE}

Salo, S., Mäkelä, J. (2016). Dyadic Emotional Interaction Style (D-EIS) - Rating scale for Marschak Interaction Method (MIM). Unpublished handbook. 